\title{
Response Characteristics of Silicon Microring Resonator Hydrogen Gas Sensor
}

\author{
Naoki Yamasaku* Non-member, Sosuke Matsuura* Non-member \\ Yoshiaki Nishijima* Non-member, Taro Arakawa* Non-member \\ Shinji Okazaki*a) Member
}

(Manuscript received April 27, 2018, revised Aug. 31, 2018)

\begin{abstract}
A novel optic sensor using platinum-loaded tungsten oxide $\left(\mathrm{Pt} / \mathrm{WO}_{3}\right)$ and a silicon microring resonator for detection of hydrogen gas at room temperature was developed and tested. The sensing principle is mainly based on the resonant wavelength shift caused by the catalytic combustion reaction of hydrogen. In a previous study, it was found that $\mathrm{Pt} / \mathrm{WO}_{3}$ film could not be deposited uniformly on the surface of the microring. To solve this problem, acetylene glycol surfactant was used and mixed with $\mathrm{Pt} / \mathrm{WO}_{3}$ precursor solution. Electron probe microanalyzer images of the device surface showed that the $\mathrm{Pt} / \mathrm{WO}_{3}$ layer successfully covered the target part of the microring. When this device was tested, a 580-pm resonant wavelength shift was observed after 20-min exposure to pure hydrogen gas at room temperature. When the previous device was tested under the same condition, a 160-pm resonant wavelength shift was observed, so the sensitivity (defined by the wavelength shift after 20-min exposure to sample gas) of the developed device was approximately four times larger than that of the previous one.
\end{abstract}

Keywords : MRR, $\mathrm{Pt} / \mathrm{WO}_{3}$, hydrogen leakage detection, sol-gel method

\section{Introduction}

Chemical sensors based on optical devices have some advantages in comparison to those based on other conventional methods, including catalytic combustion, semiconductor, and gas heat conduction. In the device component, conventional sensors have some electrodes to detect change of the electric resistance. They usually need some heating to operate properly, so it is essential to supply electrical power to them. This indicates that a system including many conventional sensors becomes too complicated and expensive. It may be possible to construct a multipoint sensor system by a wireless communication facility, but it is rather difficult to make the electrical power supply wireless. However, optic chemical sensors do not have any cable or electrode. This means that explosion-proof sensing can be relatively easily implemented. Moreover, sensing control units, including the light source to photoelectric conversion and the detector, can be completely separated from the sensing environment because the light signal is easily taken to the outside by the optical fiber. Also, a multipoint sensing system can be easily constructed by arranging sensor devices on the single optical fiber in series.

Optical fiber, which has been specially processed, is mainly used for the chemical sensor. Ke et al. reported a hydrogen sulfide gas sensor based on evanescent-wave absorption. They used a chemical-etching method to fabricate sensing probes, which function as evanescent field absorption points ${ }^{(1)}$. Korposh et al. reported an ammonia gas sensor based on evanescent-wave

a) Correspondence to: Shinji Okazaki. E-mail: okazaki-shinji-yp@ ynu.ac.jp

* Yokohama National University

79-1, Tokiwadai, Hodogaya-ku, Yokohama, Kanagawa 240-8501, Japan absorption. They prepared a multimode fiber whose exposed section of the silica core was covered with a poly(diallyldimethylammonium

chloride)/tetrakis(4-sulfophenyl)porphine bilayer ${ }^{(2)}$. Kishore et al. reported a chromium(VI) ion sensor based on a fiber Bragg grating (FBG). They coated the FBG with hydrogels. When there were chromium(VI) ions, hydrogels absorbed it and swelled, which caused mechanical strain on the $\mathrm{FBG}^{(3)}$. Tabassum et al. reported a manganese ion sensor based on surface plasmon resonance (SPR). They coated the unclad core of the multimode optical fiber with silver; subsequently, this point was covered with a $\mathrm{ZnO}_{(1-\mathrm{x})} \mathrm{PPy}_{\mathrm{x}}$ nanocomposite ${ }^{(4)}$. These studies show that, fiber-optic chemical sensors have many sensing methods.

A microring resonator (MRR) is a kind of optical waveguide that has recently attracted considerable attention for chemical or bio sensors, because the MRR is small and highly sensitive, and it is a high $\mathrm{Q}$ factor device. Moreover, it can analyze a very small amount of sensing sample ${ }^{(5)-(7)}$. Meziane et al. simulated a hexavalent chromium sensor based on evanescent-wave absorption with an MRR made of polymer. They utilized a color indicator and attempted to observe the absorbance spectrum change in the visible wavelength range ${ }^{(7)}$. Matthias Jäger et al. reported a fibrinogen sensor with a silicon-on-insulator microring resonator based on the resonant wavelength shift. They coated methylated dendric polyglycerol onto the MRR for reduction of nonspecific protein binding to the sensor surface ${ }^{(8)}$. Wang et al. demonstrated integrated microring resonator biosensors based on the resonance frequency shift. They simultaneously monitored cell growth, sodium pentachlorophenate, and Aldicar ${ }^{(9)}$. The microring resonator sensor also has multiple sensing methods. However, the above-mentioned sensors are for analytes in solution, and there are few reports for gas sensing. 
Table 1. Properties of hydrogen at $1 \mathrm{~atm}, 298 \mathrm{~K}$

\begin{tabular}{|c|c|}
\hline Property & Value \\
\hline Flammable range & $4.0-75 \mathrm{vol} . \%$ fuel in air \\
\hline Autoignition temperature & $572^{\circ} \mathrm{C}$ \\
\hline Ignition energy & $0.018 \mathrm{~mJ}$ \\
\hline Heat of combustion & $285.8 \mathrm{~kJ} / \mathrm{mol}$ \\
\hline
\end{tabular}

In this work, a silicon-on-insulator hydrogen gas sensor based on the resonant-wavelength shift is investigated. Hydrogen was selected for the measurement gas. Hydrogen has been expected to be an energy carrier in clean and sustainable energy systems, which do not emit greenhouse gases, theoretically ${ }^{(10)(11)}$. However, it has many hazardous characteristics, such as the wide flammable range and the small ignition energy (shown in Table 1). Therefore, it is essential to monitor the hydrogen concentration level properly with hydrogen sensors, especially concentrations less than the lower explosion limit (LEL) $)^{(12)(13)}$.

\section{Sensing Principle}

2.1 Microring Resonator Fig. 1 shows a schematic view of the MRR and the busline waveguides. Part of the light that enters from one side of the bus line waveguide is coupled into the MRR at a coupling region and circulates in the MRR. Subsequently, it interferes with light in the busline waveguide. As a result, transmitted light does not have a specific wavelength component, which is called "resonant wavelength". Resonant wavelength satisfies following equation.

$$
m \lambda=n_{e f f} L
$$

where $m$ is an integer, $\lambda$ is the resonant wavelength, $n_{\text {eff }}$ is the effective refractive index, and $L$ is the round-trip length of the MRR. Thus, the resonant wavelength is determined by the round-trip length of the MRR or effective refractive index of constituent materials. If the material whose refractive index is changed with respect to the hydrogen concentration or the material that expands and stretches the MRR with respect to hydrogen is utilized as the cladding of the MRR, hydrogen concentration is estimated by the resonant-wavelength shift.

2.2 Platinum-loaded Tungsten Oxide ( $\left.\mathbf{P t} / \mathrm{WO}_{3}\right) \quad \mathrm{WO}_{3}$ is well known as a gasochromic material. When it absorbs hydrogen atoms and produces tungsten bronze $\left(\mathrm{H}_{\mathrm{x}} \mathrm{WO}_{3}\right)$, its yellowish color turns deep blue. This reaction is given by this equation.

$$
(x / 2) \mathrm{H}_{2}+\mathrm{WO}_{3} \rightarrow \mathrm{H}_{x} \mathrm{WO}_{3}
$$

When $\mathrm{H}_{\mathrm{x}} \mathrm{WO}_{3}$ reacts with oxygen, it returns $\mathrm{WO}_{3}$.

$$
\mathrm{H}_{x} \mathrm{WO}_{3}+(x / 4) \mathrm{O}_{2} \rightarrow(x / 2) \mathrm{H}_{2} \mathrm{O}+\mathrm{WO}_{3}
$$

These reactions usually occur at a sufficiently high temperature. To cause them to occur even at room temperature, platinum is doped into $\mathrm{WO}_{3}$ as the catalyst. When hydrogen reaches the surface of $\mathrm{Pt}$, it is dissociated into molecules and into atoms, and, therefore, atoms spill over from the surface of $\mathrm{Pt}$ into $\mathrm{WO}_{3}$. As a result, the reaction activation energy is decreased ${ }^{(14)}$. Equations (2) and (3) are exothermic reactions, so if $\mathrm{Pt} / \mathrm{WO}_{3}$ is fixed near the MRR, it could change the effective refractive index or extend round-trip length of the MRR by the temperature rise. Moreover, the refractive index and density of this material changes while $\mathrm{WO}_{3}$ changes into $\mathrm{H}_{\mathrm{x}} \mathrm{WO}_{3}$. Therefore, if the MRR is mostly not influenced by temperature, $\mathrm{Pt} / \mathrm{WO}_{3}$ fixed on the MRR could change the effective refractive index or extend/shorten the round-trip length of the MRR.

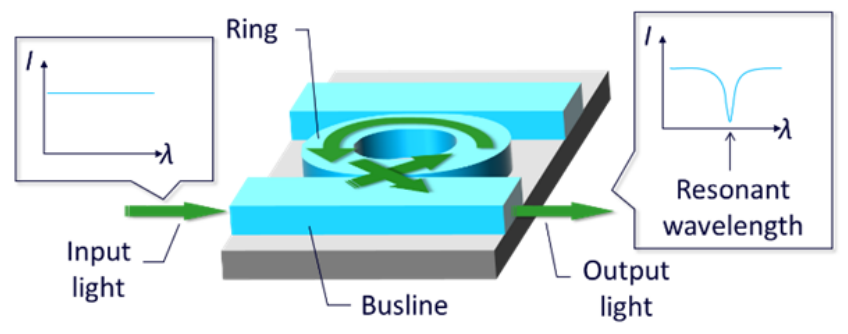

Fig. 1. Schematic view of the MRR

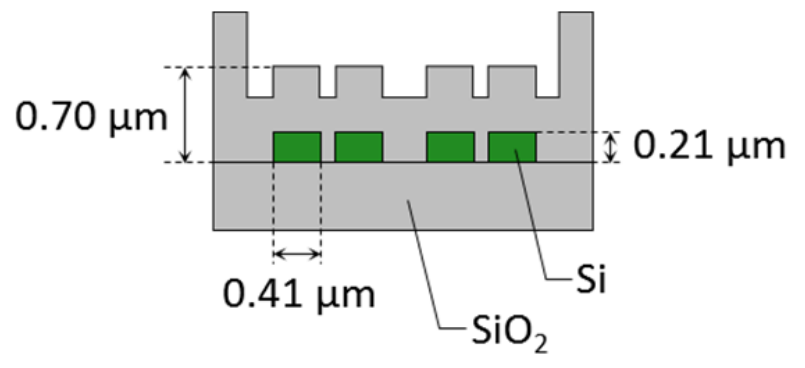

(a) Cross-sectional view of the Si MRR device

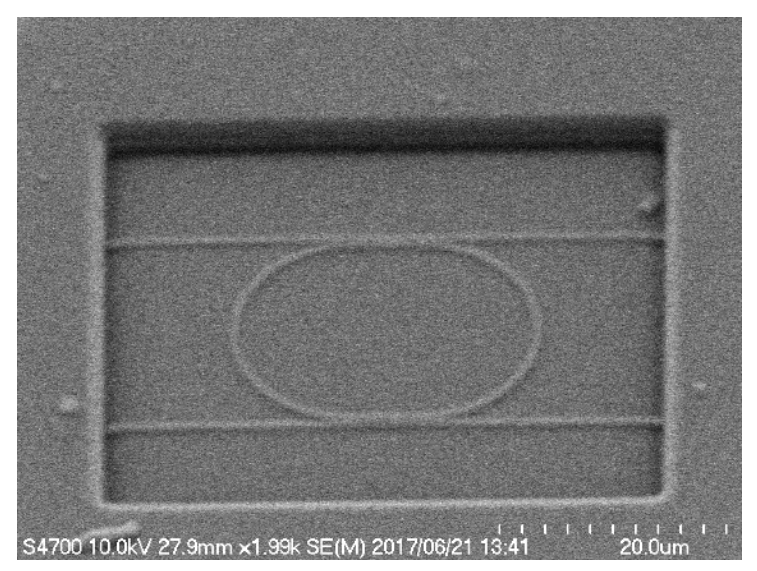

(b) SEM image of the Si MRR device

Fig. 2. Detailed images and sizes of the Si MRR device

\section{Experimental}

3.1 Fabrication of the Si MRR Device The MRR was fabricated by a complementary metal-oxide-semiconductor (CMOS)-compatible process. Fig. 2 (a) shows the cross-sectional view of the device, and Fig. 2 (b) shows the scanning electron microscopy (SEM) image of the device. To function as the gas sensor, the surface of the Si MRR was uncovered, and the other area was covered with silica as cladding. However, it was estimated that the extinction coefficient of hydrogen-sensitive film was so large that the optical signal could not be detected. Therefore, $700 \mathrm{~nm}$ of silica was sputtered onto the MRR as a buffer layer.

3.2 Preparation of $\mathbf{P t} / \mathrm{WO}_{3}$ Precursor Solution $\mathrm{Pt} / \mathrm{WO}_{3}$ was fabricated by a sol-gel method. First, $10 \mathrm{~g}$ of hydrogen hexachloroplatinate(IV) hexahydrate (FUJIFILM Wako Pure Chemical Co.) was dissolved into $25 \mathrm{~mL}$ of distilled water. $500 \mu \mathrm{L}$ of this solution was dissolved into $4.50 \mathrm{~mL}$ of ethanol aq. Then, 1 vol.\% acetylene glycol surfactant (Surfynol 465, Nissin Chemical Industry Co.) was dropped into it and stirred for more than $30 \mathrm{~min}$. For convenience, it was named Solution A. Next, 0.5-M sodium tungstate aq. was prepared by dissolving sodium tungstate(VI) dihydrate (FUJIFILM Wako Pure Chemical Co.) into distilled 


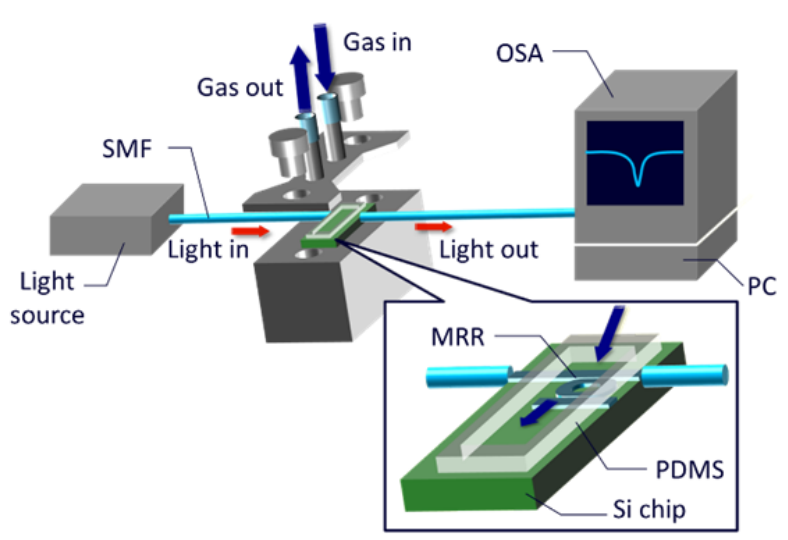

Fig. 3. Gas-sensing apparatus

water. To gain 0.5-M tungstic acid aq., it was ion-exchanged with a hydrogen ion type of cation exchange resin (IR120BH, Organo Co.). Then, $3.25 \mathrm{~mL}$ of tungstic acid aq. was dissolved into Solution $\mathrm{A}$ and stirred. As a result, a precursor solution of $\mathrm{Pt} / \mathrm{WO}_{3}$ was prepared.

3.3 Deposition The Si MRR device was spin-coated with the precursor solution. The spin rate was $500 \mathrm{rpm}$ and the spin time was $300 \mathrm{~s}$. It was desiccated in the desiccator for $60 \mathrm{~min}$ and subsequently calcined in the furnace for $60 \mathrm{~min}$ at $500^{\circ} \mathrm{C}$. After calcination, it was cooled to room temperature in the furnace. Before gas-sensing tests, The Q factor of this was calculated. It was calculated by the full-width half-maximum (3-dB bandwidth) divided by the resonant wavelength, and the value was 3520.455 .

3.4 Gas-sensing Apparatus To test the property of the fabricated device, the gas-sensing system was custom designed. Fig. 3 shows the schematic image of this. This chamber was static. A $\mathrm{He}-\mathrm{Ne}$ laser whose wavelength was $632.8 \mathrm{~nm}$ was utilized for optical-axis alignment. Each side of the sensing device was connected to the single-mode optic fibers with diameters of $900 \mu \mathrm{m}$. One of the fibers and the infrared light source were coupled with the lens. The light induction was operated with the spot size converter. The other connected it to the optical spectrum analyzer. The flow path was formed on the sensing device with polydimethylsiloxane. Sample gas was allowed to flow through the flow path with the micro syringe. The Peltier device was attached to the sensing apparatus and kept at room temperature. The sensing test was performed in atmospheric pressure at room temperature.

\section{Results and Discussion}

4.1 Surface of the Fabricated Devices Fig. 4. Shows the SEM images of each fabricated device. The image of the device whose hydrogen-sensitive film was deposited without containing surfactant into the precursor solution is shown in Fig. 4 (a). The surface of the device, except the part of the MRR, is obviously covered with $\mathrm{Pt} / \mathrm{WO}_{3}$, but the MRR is hardly covered. However, the one whose film was deposited containing surfactant into the precursor is shown in Fig. 4 (b). Not only the surface of the device around the MRR but also the MRR itself are sufficiently covered with $\mathrm{Pt} / \mathrm{WO}_{3}$. These results indicate that the surfactant reduced the surface tension of the precursor and allowed it to wet the part of the MRR that had an intricate structure. Fig. 5 shows the result of the electron probe microanalyzer (EPMA) analysis of the device shown in Fig. 4 (b). Tungsten and platinum signals were gained

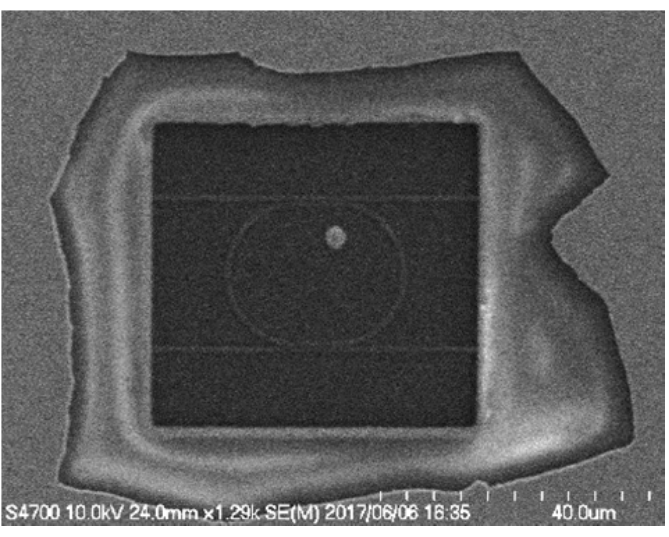

(a) Fabricated without surfactant

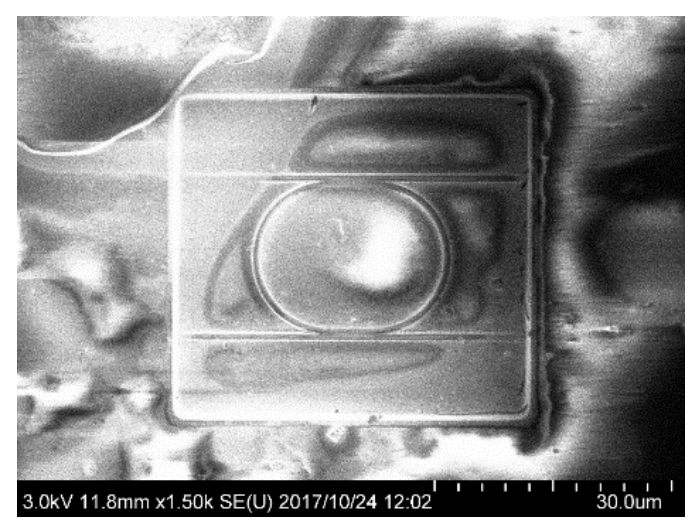

(b) Fabricated with surfactant

Fig. 4. SEM images of the surface of the fabricated sensor devices
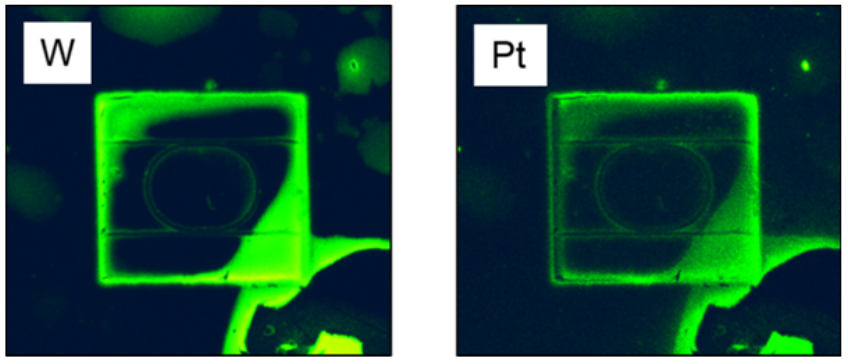

Fig. 5. EPMA images of the sensor device

along the waveguide. It is assumed that the $\mathrm{Pt} / \mathrm{WO}_{3}$ film may function as a cladding layer.

4.2 XRD Analyses of the $\mathbf{P t} / \mathrm{WO}_{3}$ Film The precursor solution with/without surfactant was used, and $\mathrm{Pt} / \mathrm{WO}_{3}$ film was deposited onto each quartz glass substrate. Subsequently, they were analyzed, and Fig. 6. shows the results of the x-ray diffraction (XRD) analyses. Fig. 6 (a) shows the Pt/ $\mathrm{WO}_{3}$ film fabricated without surfactant, whereas Fig. 6 (b) shows the one fabricated with surfactant. Comparing Figs. 6 (a) with (b), it is indicated that the crystallinity of $\mathrm{WO}_{3}$, especially the plane orientation (110), was improved by the surfactant. In both results, any peaks related to Pt, which certainly is contained in the film, were not observed. This reason is assumed to be that Pt exists in microcrystalline form and is well distributed in the film. In addition, in Fig. 6 (b), peaks related to the surfactant were also not observed. The reason is assumed to be that the surfactant was burnt out during calcination rather than well distributed in the film in microcrystalline form. 


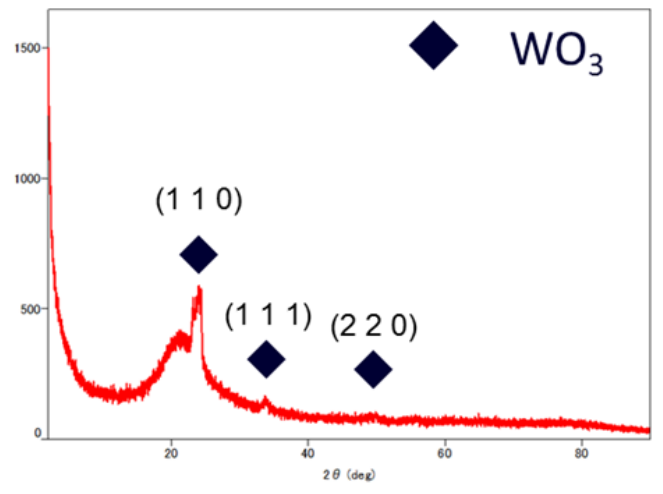

(a) Fabricated without surfactant

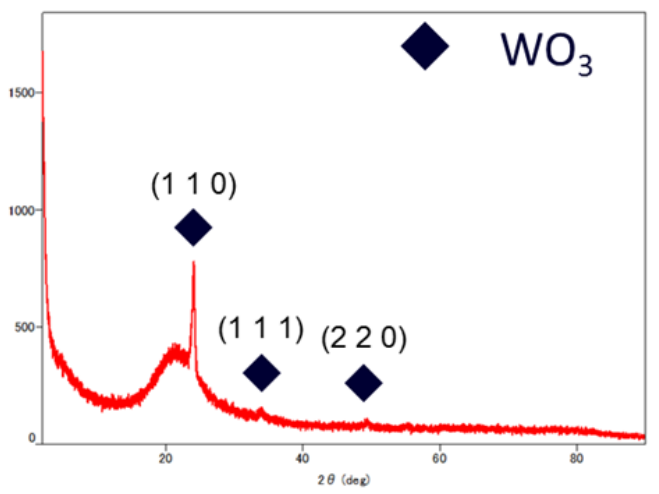

(b) Fabricated with surfactant

Fig. 6. XRD patterns of the fabricated $\mathrm{Pt} / \mathrm{WO}_{3}$

\subsection{Test of the Temperature Sensitivity of the Device}

To test the temperature sensitivity of the device, resonant wavelength shifts corresponding to temperature change were obtained. The Peltier device was utilized to control the temperature of the experimental system. Fig. 7 shows the results. They show that this device corresponds to the temperature change well and linearly, and there is a significant difference between before $\mathrm{Pt} / \mathrm{WO}_{3}$ with surfactant coating and after coating. The latter is more sensitive to temperature change than the former. It is assumed that this is because the thermal expansion coefficient of the $\mathrm{Pt} / \mathrm{WO}_{3}$ film is so much larger than that of the MRR that the MRR is stretched by $\mathrm{Pt} / \mathrm{WO}_{3}$, and, as a result, the round-trip length of the MRR becomes longer more rapidly. Mishra et al. have attempted to improve the temperature sensitivity of FBG by coatings of different materials ${ }^{(15)}$, and the theory of improvement agrees with the above-mentioned considerations.

4.4 Hydrogen Gas Sensing Fig. 8 shows the results with static exposure to pure hydrogen gas at room temperature. The resonant wavelength of the sensor fabricated without surfactant shifted by approximately $160 \mathrm{~nm}$ after $20 \mathrm{~min}$ exposure to pure hydrogen. However, the one fabricated with surfactant shifted by approximately $580 \mathrm{~nm}$. It was nearly four times larger than that fabricated without surfactant. This difference is because the MRR of the latter was not so covered with $\mathrm{Pt} / \mathrm{WO}_{3}$ that it could not respond to hydrogen efficiently. However, the one fabricated without surfactant slightly responded to hydrogen. This indicates that, because the reactions represented as the reaction formulae in Equations (2) and (3) were exothermic, the MRR responded to the temperature change indirectly from $\mathrm{Pt} / \mathrm{WO}_{3}$ located at a small distance. Fig. 9 shows the behavior of the sensor fabricated with surfactant in different atmospheres $\left(\mathrm{N}_{2}\right.$ and air) after static

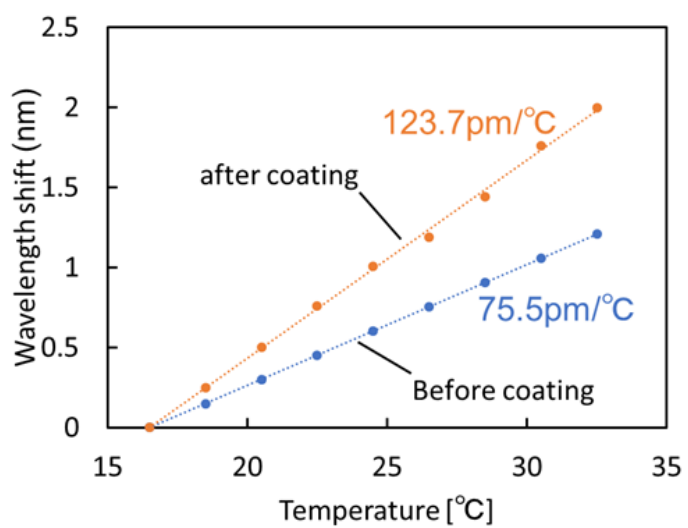

Fig. 7. Temperature sensitivity of the sensing device

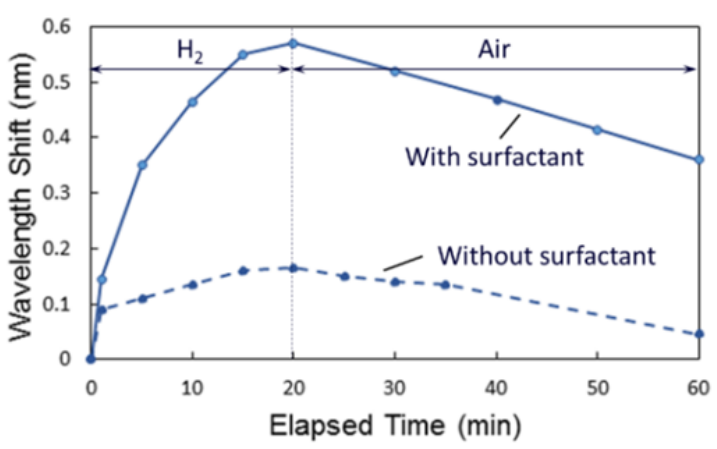

Fig. 8. Sensor responses to pure hydrogen

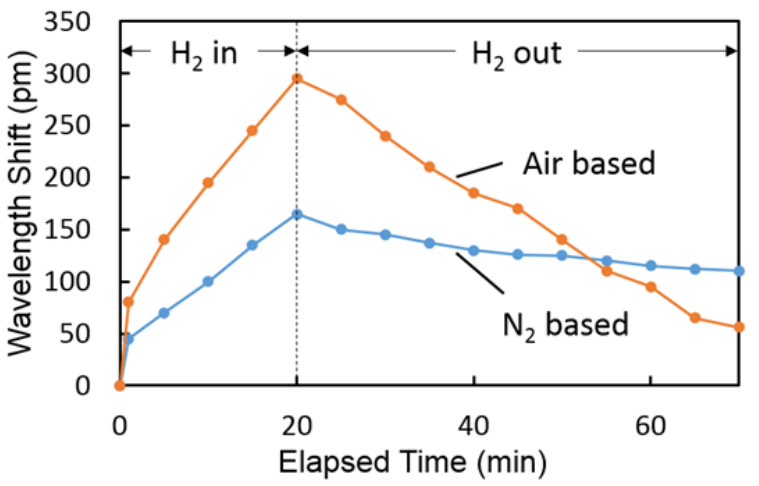

Fig. 9. Sensor behaviors in different atmosphere

exposure to pure hydrogen at room temperature. Compared with Fig. 8, it seems that the rise time of the resonant wavelength shift is different. This is because the MRR chip of the sensor is different from the one used in Fig. 8. As shown, the resonant wavelength shift of the sensor in $\mathrm{N}_{2}$ significantly remained, whereas that in air almost recovered. In $\mathrm{N}_{2}$, the reaction represented as Equation (3) does not occur, so tungsten bronze $\left(\mathrm{H}_{\mathrm{x}} \mathrm{WO}_{3}\right)$ still exists. The refractive index of $\mathrm{H}_{\mathrm{x}} \mathrm{WO}_{3}$ is clearly different from that of $\mathrm{WO}_{3}$. Therefore, it is surmised that the resonant wavelength shift corresponds to not only the thermal expansion, but also to the change in the effective refractive index of the cladding, or the strain induced by the change of $\mathrm{WO}_{3}$ into $\mathrm{H}_{\mathrm{x}} \mathrm{WO}_{3}$ also affects the resonant-wavelength shift. Unfortunately, it is not clear what factor is dominant among the sensor characteristics from these results. 


\section{Conclusion}

In this study, an MRR gas sensor based on the resonant wavelength shift is proposed utilizing gasochromic material. When sol-gel method was selected, reducing the surface tension of the precursor solution of the hydrogen-sensitive material is the key point determining whether the fabricated device functions well. In addition, it is surmised that the resonant-wavelength shift corresponds to not only the thermal expansion, but also the change in the effective refractive index of the cladding and the strain induced by the change of $\mathrm{WO}_{3}$ into $\mathrm{H}_{\mathrm{x}} \mathrm{WO}_{3}$. Further investigation is needed to reveal what factor is dominant among the sensor characteristics.

\section{Acknowledgment}

The staff of the Instrumental Analysis Center of Yokohama National University are gratefully acknowledged.

\section{References}

(1) Z.-J. Ke, D.-L. Tang, X. Lai, Z.-Y. Dai, and Q. Zhang : "Optical fiber evanescent-wave sensing technology of hydrogen sulfide gas concentration in oil and gas fields", Optik, Vol.157, pp.1094-1100 (2018)

(2) S. Korposh, S. Kodaira, R. Selyanchyn, F. H. Ledezma, S. W. James, and S.-W. Lee : "Porphyrin-nanoassembled fiber-optic gas sensor fabrication: Optimization of parameters for sensitive ammonia gas detection", Optics and Laser Technology, Vol.101, pp.1-10 (2018)

(3) P. V. N. Kishore, M. S. Shankar, and M. Satyanarayana: "Detection of trace amounts of chromium(VI) using hydrogel coated Fiber Bragg grating", Sensors and Actuators B: Chemical, Vol.243, pp.626-633 (2017)

(4) R. Tabassum and B. D. Gupta : "Fiber optic manganese ions sensor using SPR and nanocomposite of ZnO-polypyrrole", Sensors and Actuators B: Chemical, Vol.220, pp.903-909 (2015)

(5) D. Dai and S. He : "Highly-sensitive sensor with large measurement range realized with wo cascaded-microring resonators", Optics Communications, Vol.279, pp.89-93 (2007)

(6) T. Ling, S.-L. Chen, and L. J. Guo : "High-sensitivity and wide-directivity ultrasound detection using high Q polymer microring resonators", APPLIED PHYSICS LETTERS, Vol.98, pp.204103 (2011)

(7) F. Meziane, V. Raimbault, H. Hallil, S. Joly, V. Conédéra, J. L. Lachaud, L. Béchou, D. Rebière, and C. Dejous : "Study of a polymer optical microring resonator for hexavalent chromium sensing", Sensors and Actuators B: Chemical, Vol.209, pp.1049-1056 (2015)

(8) M. Jäger, T. Becherer, J. Bruns, R. Haag, and K. Petermann : "Antifouling coatings on SOI microring resonators for bio sensing applications", Sensors and Actuators B: Chemical, Vol.223, pp.400-405 (2015)

(9) S. Wang, A. Ramachandran, and S. Ja : "Integrated microring resonator biosensors for monitoring cell growth and detection of toxic chemicals in water", Biosensors and Bioelectronics, Vol.24, pp.3061-3066 (2009)

(10) S. Dunn : "Hydrogen futures: towards a sustainable energy system", International Journal of Hydrogen Energy, Vol.27, pp.235-264 (2002)

(11) M. Momirlan and T. N. Veziroglu : "The properties of hydrogen a fuel tomorrow in sustainable energy system for a cleaner planet", International Journal of Hydrogen Energy, Vol.30, pp.795-802 (2005)

(12) D. A. Crowl and Y.-D. Jo : "The hazards and risks of hydrogen", Journal of Loss Prevention in the Process Industries, Vol.20, pp.158-164 (2007)

(13) V. A. Petuknov, I. M. Naboko, and V. E. Fortov : "Explosion hazard of hydrogen-air mixtures in the large volumes", International Journal of Hydrogen Energy, Vol.34, pp.5924-5931 (2009)

(14) Y. Zhang, H. Peng, X. Qian, Y. Zhang, G. An, and Y. Zhao : "Recent advancements in optical fiber hydrogen sensors", Sensors and Actuators B: Chemical, Vol.244, pp.393-416 (2017)

(15) V. Mishra, M. Lohar, and A. Amphawan : "Improvement in temperature sensitivity of FBG by coating of different materials", Optik, Vol.127, pp.825-828 (2016)

Naoki Yamasaku (Non-member) received a B.S. degree in the

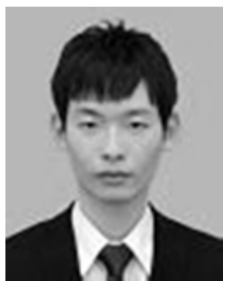

Department of Chemistry, Chemical Engineering and Life Science from Yokohama National University, Japan in 2016. He is currently working towards an M.S. degree in the Department of Materials Science and Engineering, Yokohama National University, Japan. His interest is in chemical sensors. He is a student member of the Electrochemical Society of Japan.

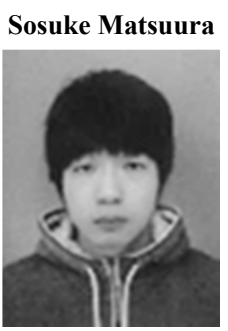

Physics.

Yoshiaki Nishijima (Non-member) received a Ph.D. degree in

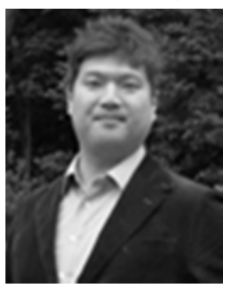
information science from Hokkaido university, Japan in 2009. He was a research fellow (DC2, PD) of Japan Society for the Promotion of Science during 2008 to 2010. He was also a doctoral research fellow of the Research Institute for Electronic Science Hokkaido University during 2010 to 2011. He joined the Department of Physics, Electrical and Computer Engineering, Yokohama National University, Japan, as an assistant professor and became an associate professor in 2013. His interests are in optical nanomaterial, nanophotonics, plasmonics, and sensors.

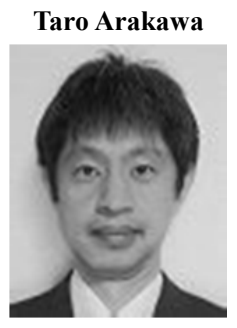

(Non-member) received a Ph.D. degree in engineering from the University of Tokyo, Japan in 1992. He joined the Department of physics, Electrical and Computer Engineering, Yokohama National University, Japan, as an assistant and became a lecturer, an assistant professor, and subsequently an associate professor in 2007. His interests are in semiconductors and optoelectronics. He is a member of IEEE, the Japan Society of Applied Physics, and the Institute of Electronics, Information and Communication Engineers.

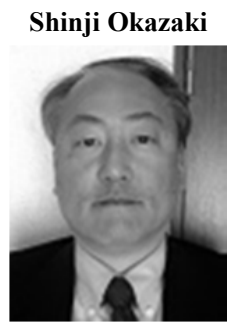

(Member) received a Ph.D. degree in the Faculty of Engineering from Yokohama National University, Japan in 2004. He joined Yokohama National University as an associate professor in 2004 and became a professor in 2015. His interests are in electrochemistry and sensor engineering. $\mathrm{He}$ is a member of the Electrochemical Society of Japan, Japan Society for Safety Engineering, and High Pressure Institute of Japan. 